\title{
Successful allotransplantation of cryopreserved tracheal grafts with preservation of the pars membranacea in nonhuman primates
}

\author{
Tomohiro Murakawa, MD \\ Jun Nakajima, MD \\ Noboru Motomura, MD \\ Arata Murakami, MD \\ Shinichi Takamoto, MD
}

From the Department of Cardiothoracic Surgery, Faculty of Medicine, University of Tokyo, Tokyo, Japan.

Received for publication March 26, 2001; revisions requested May 13, 2001; revisions received July 17, 2001; accepted for publication July 23, 2001.

Address for reprints: Tomohiro Murakawa, MD, Department of Cardiothoracic Surgery, Faculty of Medicine, University of Tokyo, 7-3-1 Hongo, Bunkyo-ku, Tokyo 113-8655, Japan (E-mail: MURAKAWA-THO@h.utokyo.ac.jp or murakawa-tky@umin.ac.jp).

J Thorac Cardiovasc Surg 2002;123:153-60

Copyright (C) 2002 by The American Association for Thoracic Surgery

$0022-5223 / 2002 \$ 35.00+0 \quad \mathbf{1 2 / 1 / 1 1 9 0 5 6}$

doi:10.1067/mtc.2002.119056

Objective: This study was performed to confirm the feasibility of cryopreserved tracheal allotransplantation in primates, the anatomy and immunology of which are considered to be more closely related to those of humans than those of other animals.

Methods: Cryopreserved tracheal allotransplantations were performed in 3 recipient primates. In the control group fresh tracheal allotransplantations were performed in 2 primates (control A), and a tracheal allotransplantation with a simply frozen tracheal graft was performed in 1 primate (control B). Monthly bronchoscopic examinations, histologic examinations, electron microscopic examinations, and immunohistochemical investigations were performed in each of the primates.

Results: In the cryopreserved tracheal allotransplantation group, 3 recipient monkeys were killed on the 35th, 144th, and 387th postoperative days, respectively. All grafts were incorporated by the recipient trachea without stenosis in the cryopreserved group. In the control group 2 recipient monkeys were killed on the 93rd postoperative day (control A), and one was killed on the 84th postoperative day (control B). Severe stenosis was observed after the transplantation in all of the control monkeys. Immunologic reactions appeared to be attenuated by the cryopreservation, whereas $\mathrm{T}$ cell-mediated immunologic rejection (control A) and loss of cartilage viability (control B) were considered to be the causes of graft failure in the control group.

Conclusion: The immunogenicity of the tracheal allografts was reduced by cryopreservation, and cryopreserved tracheal allotransplantation was successful in our primate model. Further investigation of cryopreserved tracheal allotransplantation with regard to proper clinical applications and the limitations of the procedure should be performed.

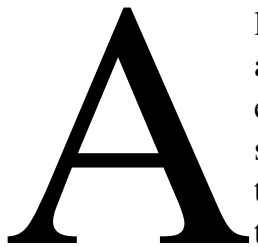

lthough the possible clinical application of cryopreserved tracheal allotransplantation has been investigated in various animal models, such as dogs and rats, with encouraging results, ${ }^{1-5}$ there is still some concern regarding interspecies differences in immunoreactions, tissue remodeling, anatomy, and so on. Moreover, the history of cryopreserved cardiac valve allotransplantation suggests that immunologic rejection is inevitable. ${ }^{6}$ Improvements in tissue cryopreservation techniques have made it possible to supply cryopreserved tissues when there is a clinical demand for them, ${ }^{7}$ and many experiments have shown that the immunoreactivity of cryopreserved tracheal allografts is less potent than that of fresh allografts. ${ }^{4,5,8}$ Although some researchers have commented that the clinical applications of cryopreserved tracheal allotransplantations might be limited, ${ }^{9-13}$ the potential usefulness of cryopreserved tracheal allografts in critical situations, such 

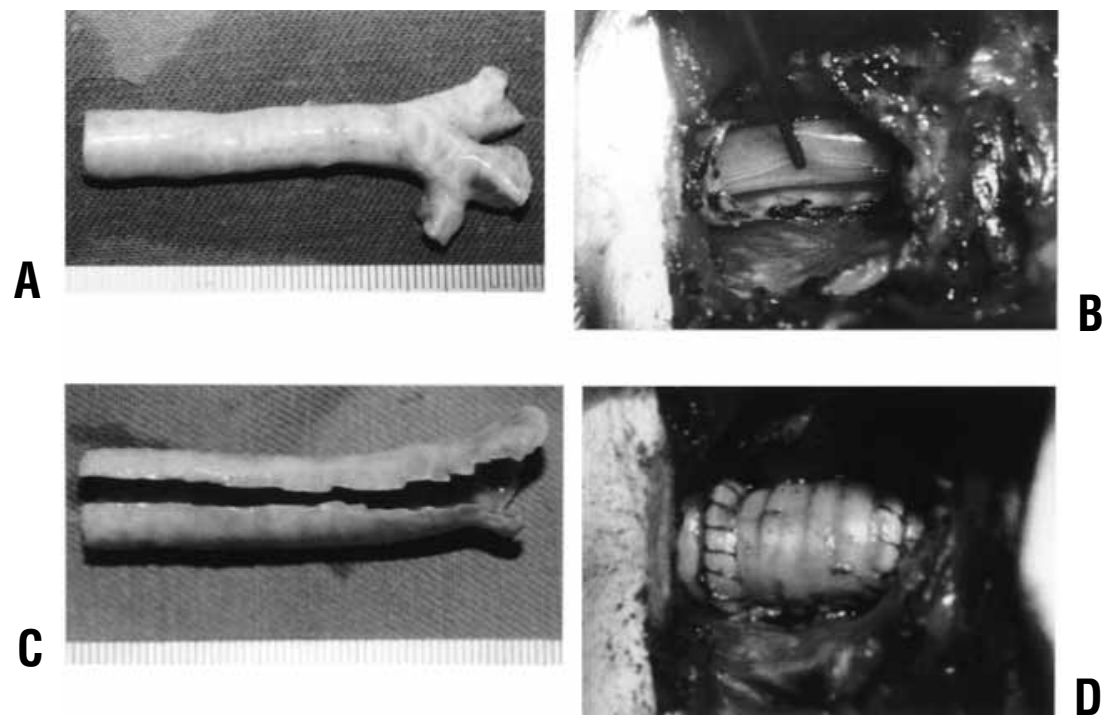

Figure 1. Preparations of cryopreserved tracheal allografts and operative procedure. A cryopreserved tracheal allograft was prepared (A). Five rings of the recipient trachea were resected with the membranous portion of the graft left intact (B), and a cryopreserved tracheal allograft was transplanted after the resection of the membranous portion (C). The donor trachea was orthotopically transplanted (D).

as congenital long-segment tracheal stenosis, should be fully investigated. Cryopreserved tracheal allografts may be applied in critical situations, such as tracheal stenosis, as has been shown by Jacobs and colleagues ${ }^{14}$ and Elliot and coworkers ${ }^{15}$ using chemically preserved allografts and by Kunachak and colleagues using irradiated cryopreserved allografts. ${ }^{16}$

This study was designed to examine the feasibility of using cryopreserved tracheal allografts in clinical applications. Primates were selected as our animal model of choice from the viewpoint of anatomy and the close immunologic relationship between primates and human beings.

\section{Materials and Methods \\ Humane Animal Care}

Special humane animal-care measures were taken in compliance with the "Guide for Animal Experimentation, Faculty of Medicine, University of Tokyo, revised 1996" and the "Guide for the Care and Use of Laboratory Animals" prepared by the Institute of Laboratory Animal Resources and published by the National Institute of Health (National Institutes of Health publication No. 85-23, revised 1985). The number of animals was limited to the minimum number necessary to obtain useful results.

\section{Preparation of Cryopreserved Tracheal Allografts}

After killing the donor primates (Japanese monkey: Macaca fuscata), the tracheas were dissected under sterile conditions. After immersing the tracheas in Roswell Park Memorial Institute (RPMI) medium 1640 (\#31800-022; Gibco BRL, Life Technologies, Inc) containing 15\% fetal bovine serum (\#26140079, Gibco BRL) and 4 kinds of antibiotics (cefmetazole sodium,
$240 \mu \mathrm{g} / \mathrm{mL}$; lincomycin hydrochloride, $120 \mu \mathrm{g} / \mathrm{mL}$; vancomycin hydrochloride, $50 \mu \mathrm{g} / \mathrm{mL}$; and polymixin B sulfate, $1000 \mathrm{U} / \mathrm{mL}$ ) for 24 hours at $4^{\circ} \mathrm{C}$, the tracheas were conjugated in RPMI medium 1640 containing $20 \%$ fetal bovine serum and $10 \%$ dimethylsulfoxide (\#134-06, Nacalai Tesque Inc) and then frozen in a programmable freezer (Profreezer, NipponFreezer Co). The following freezing program was used: the specimens were cooled from room temperature to $4^{\circ} \mathrm{C}$ at a rate of $3^{\circ} \mathrm{C} / \mathrm{min}$ and then maintained at $4^{\circ} \mathrm{C}$ for 5 minutes. They were then cooled at $1^{\circ} \mathrm{C} / \mathrm{min}$ from $4^{\circ} \mathrm{C}$ to $-50^{\circ} \mathrm{C}$ and at $5^{\circ} \mathrm{C} / \mathrm{min}$ from $-50^{\circ} \mathrm{C}$ to $-80^{\circ} \mathrm{C}$. After the temperature of the specimens reached $-80^{\circ} \mathrm{C}$, they were preserved at $-180^{\circ} \mathrm{C}$ in the vapor phase of liquid nitrogen for 9 to 12 months. The grafts were thawed rapidly to $37^{\circ} \mathrm{C}$ and immersed in RPMI medium 1640 containing $15 \%$ fetal bovine serum before transplantation (Figure 1, A). A histologic evaluation of fresh and cryopreserved tracheas was performed afterward.

\section{Operative Procedure}

Recipient primates (Japanese monkey: Macaca fuscata) were anesthetized with ketamine and sodium pentobarbital intramuscularly. The primates were intubated orally with a 5-mm endotracheal tube and ventilated with a MARK7 ventilator (Bird Corp). All animals were placed in a supine position. A median sternotomy and a median cervical incision were made, and the cervical and upper mediastinal tracheas were exposed without impairing the vessels around the trachea. A 5-ring length of recipient trachea was resected, leaving the membranous portion of the trachea intact (Figure 1,B). The tip of the endotracheal tube was placed distal to the distal cut end of the recipient trachea, and ventilation was maintained throughout the procedure. After rapid thawing of the cryopreserved donor trachea, the membranous portion of the donor trachea was resected (Figure 1,C). A 7-ring 

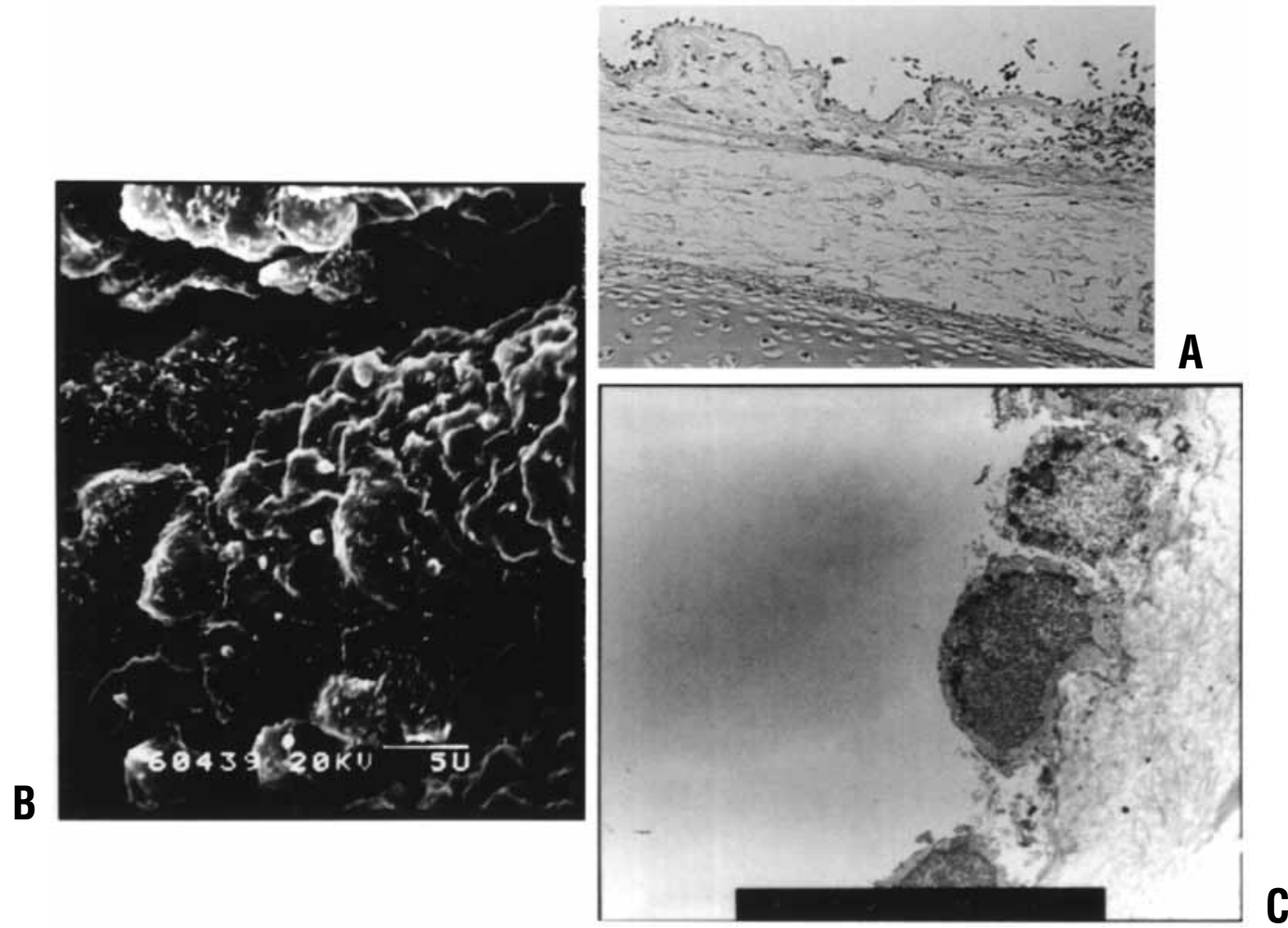

Figure 2. Histologic and electron microscopic findings after graft thawing. After the cryopreservation and thawing procedure, the major histologic change was the detachment of the airway epithelium ( $A$ and $B$ ). The intracellular organelles in the residual epithelium were mostly destroyed (C). The cartilage was well preserved, even after 12 months of cryopreservation (A). A, Hematoxylin-and-eosin staining, original magnification $\times 10$; B, scanning electron microscopy, original magnification $\times 2400$; C, transmission electron microscopy, original magnification $\times 6000$.

length of the donor trachea was then transplanted by means of continuous suturing with 5-0 PDS II (Z863E; Ethicon, Johnson \& Johnson Int, Brussels, Belgium), and the recipient airway was reconstructed without impairing the patency of the airway (Figure $1, D)$. The first and last rings of the proximal and distal end of the donor trachea were used as suturing cuffs. Thus the 5-ring length of recipient trachea was reconstructed with a cryopreserved allograft. A pedicled omental flap was not used. After full recovery from anesthesia, the recipient monkeys were extubated and transferred to a recovery room. Careful postoperative care, especially with regard to airway clearance, was taken. A prophylactic daily intramuscular injection of $1 \mathrm{~g}$ of ampicillin was performed until the seventh postoperative day to prevent postoperative mediastinitis. No immunosuppressants were given at any time during the course of the experiment. Cryopreserved tracheal allotransplantations were performed in 3 monkeys.

\section{Study Control}

Several experiments have shown that cryopreservation attenuates the allogenicity of tracheal allografts, ${ }^{4,5,8}$ and cryopreservation with a programmable freezer is believed to increase the tissue viability of cryopreserved allografts. ${ }^{7}$ To confirm whether these findings are applicable in primates, we performed fresh tracheal allotransplantations in 2 recipient monkeys immediately after the removal of the tracheas from the donor monkeys (control A). We also performed a simple frozen tracheal allotransplantation in 1 recipient monkey with a donor trachea that was conjugated in RPMI medium 1640 without dimethylsulfoxide and immediately frozen in liquid nitrogen without using a programmable freezer (control B). The operative procedure for the study control groups was the same as that for the programmed-frozen tracheal allotransplantations.

\section{Postoperative Evaluations}

Monthly flexible fiberoptic bronchoscopies were performed after the tracheal allotransplantations to observe changes within the trachea. During chronic periods, the recipient monkeys were killed, and the transplanted grafts were removed en bloc for pathologic examination. Histologic changes in the allotransplanted tracheas were examined with hematoxylin-and-eosin staining, immunohistochemical staining, scanning electron microscopy, and transmission electron microscopy. Histologic changes in the tracheas during the preparation of the tracheas were also examined.

Immunostaining reactions for $\mathrm{CD} 3$, major histocompatibility complex (MHC) class II antigen, and S100 protein were detected with the DAKO catalyzed signal amplification system (K1500, $\mathrm{DAKO}$ ) for each 5- $\mu \mathrm{m}$ section. The primary antibodies were antiCD3 antibody (A0452, DAKO A/S) diluted 1:50, anti-MHC class II antibody (M0775, DAKO A/S) diluted 1:50, and anti-S100 protein antibody (Z0311, DAKO A/S) diluted 1:1000. 

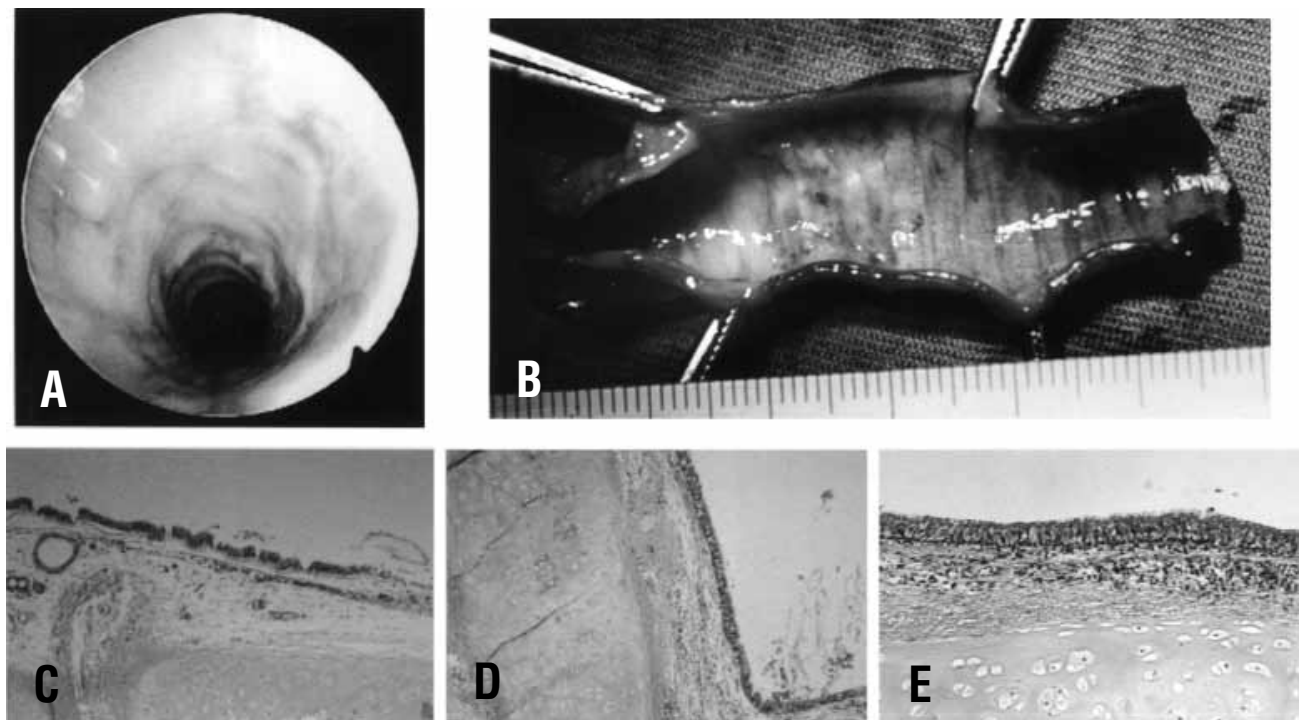

Figure 3. Bronchoscopic, macroscopic, and histologic findings of the cryopreserved tracheal allotransplantation group. Bronchoscopic findings in the cryopreserved tracheal allotransplantation group revealed that grafts were well incorporated, with good airway patency and a good airway epithelium lining (A). Macroscopic and histologic examination of the transplanted grafts in the cryopreserved allotransplantation group revealed a good patency of the implanted grafts with dense epithelial lining and well-maintained cartilaginous viability (B-E). A small degree of intercartilaginous tissue shrinkage was observed (C), and a mild degree of mononuclear cell infiltration was observed in the submucosal layer (E). This Figure represents findings 12 months after transplantation. Hematoxylin-and-eosin staining: $C$ and $D$, originally $\times 4 ; E$, originally $\times 10$.

TABLE 1. Results for the cryopreserved tracheal allotransplantation group

\begin{tabular}{lllccrr}
\hline No. & Recipient & \multicolumn{1}{c}{ Donor } & Preservation period (d) & Status of graft & Survival (d) & Cause of death \\
\hline $1^{*}$ & $7.0 \mathrm{~kg}$, male & $8.0 \mathrm{~kg}$, male & 367 & Incorporated & 35 & Killed \\
$2^{*}$ & $8.6 \mathrm{~kg}$, male & $7.6 \mathrm{~kg}$, female & 282 & Incorporated & 144 & Killed \\
$3^{*}$ & $7.6 \mathrm{~kg}$, female & $8.0 \mathrm{~kg}$, male & 302 & Incorporated & 387 & Killed \\
\hline
\end{tabular}

${ }^{*}$ Donor tracheas were frozen in a programmable freezer and preserved in liquid nitrogen for 9 to 12 months.

TABLE 2. Results for the control group

\begin{tabular}{lcccccc}
\hline No. & Recipient & Donor & Preservation period (d) & Status of graft & Survival (d) & Cause of death \\
\hline $4^{*}$ & $11.2 \mathrm{~kg}$, male & $9.0 \mathrm{~kg}$, male & - & Severe stenosis & 93 & Killed \\
$5^{*}$ & $9.0 \mathrm{~kg}$, male & $11.2 \mathrm{~kg}$, male & - & Severe stenosis & 93 & Killed \\
$6^{*}$ & $5.5 \mathrm{~kg}$, female & $8.3 \mathrm{~kg}$, male & 36 & Severe stenosis & 84 & Killed \\
\hline
\end{tabular}

*Fresh tracheal allotransplantations were performed in monkeys 4 and 5, and a simply frozen trachea was allotransplanted in monkey 6.

\section{Results}

The most remarkable change produced by the cryopreservation and thawing procedure was the exfoliation of airway epithelial cells in the tracheal allograft (Figure 2, $A$ and $B$ ). After the detachment of the airway epithelium, the basal membrane directly exposed the luminal surface of the graft (Figure 2, $A$ and $B$ ), and the intracellular organelles of the residual epithelium were mostly destroyed (Figure 2, $C$ ). The structure of the cartilaginous rings was well preserved during the cryopreservation and thawing procedure (Figure 2, A).
In the cryopreserved tracheal allotransplantation group (Table 1), all the primates survived the procedure. Although bronchoscopic examination during the early postoperative period revealed mucosa sloughing of the transplanted graft and slight sputum retention, probably caused by the loss of the tracheal epithelium during the cryopreservation and thawing procedure, these findings disappeared within 1 month. Recovery was uneventful, with complete patency of the transplanted allografts (Figure 3, $A$ and $B$ ). A small degree of intercartilaginous tissue shrinkage was observed 


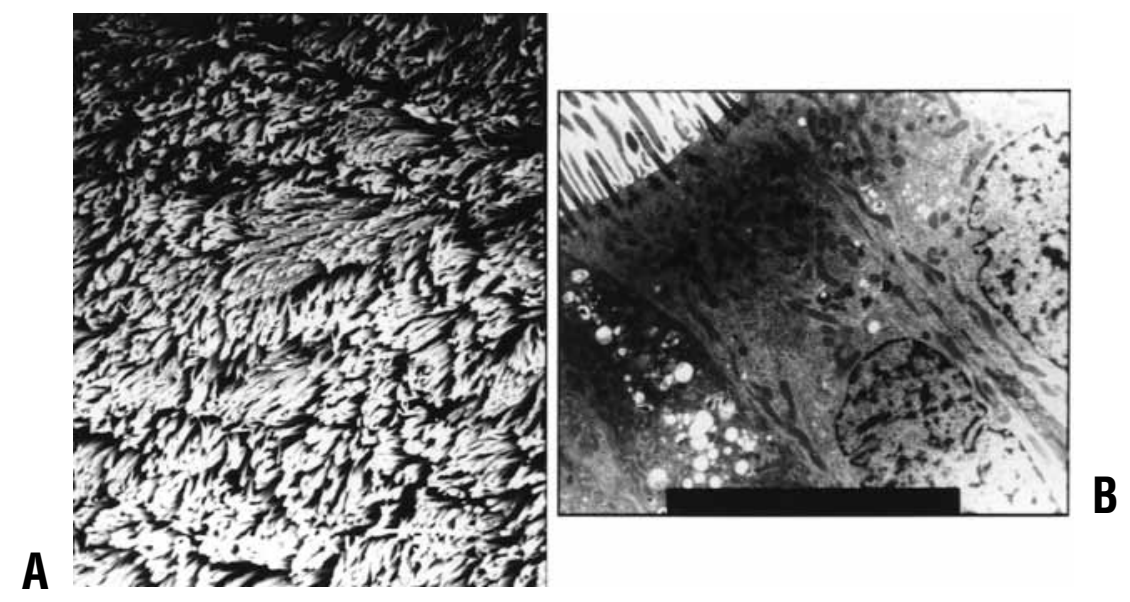

Figure 4. Electron microscopic findings of the cryopreserved tracheal allotransplantation group. Electron microscopic examination revealed a dense covering of airway epithelial cells on the implanted grafts $(A)$ and well-developed intracellular organelles, indicating good cellular function (B). This Figure represents findings 12 months after transplantation. A, Scanning electron microscopy; original magnification $\times 2400$; B, transmission electron microscopy, original magnification $\times 6000$.

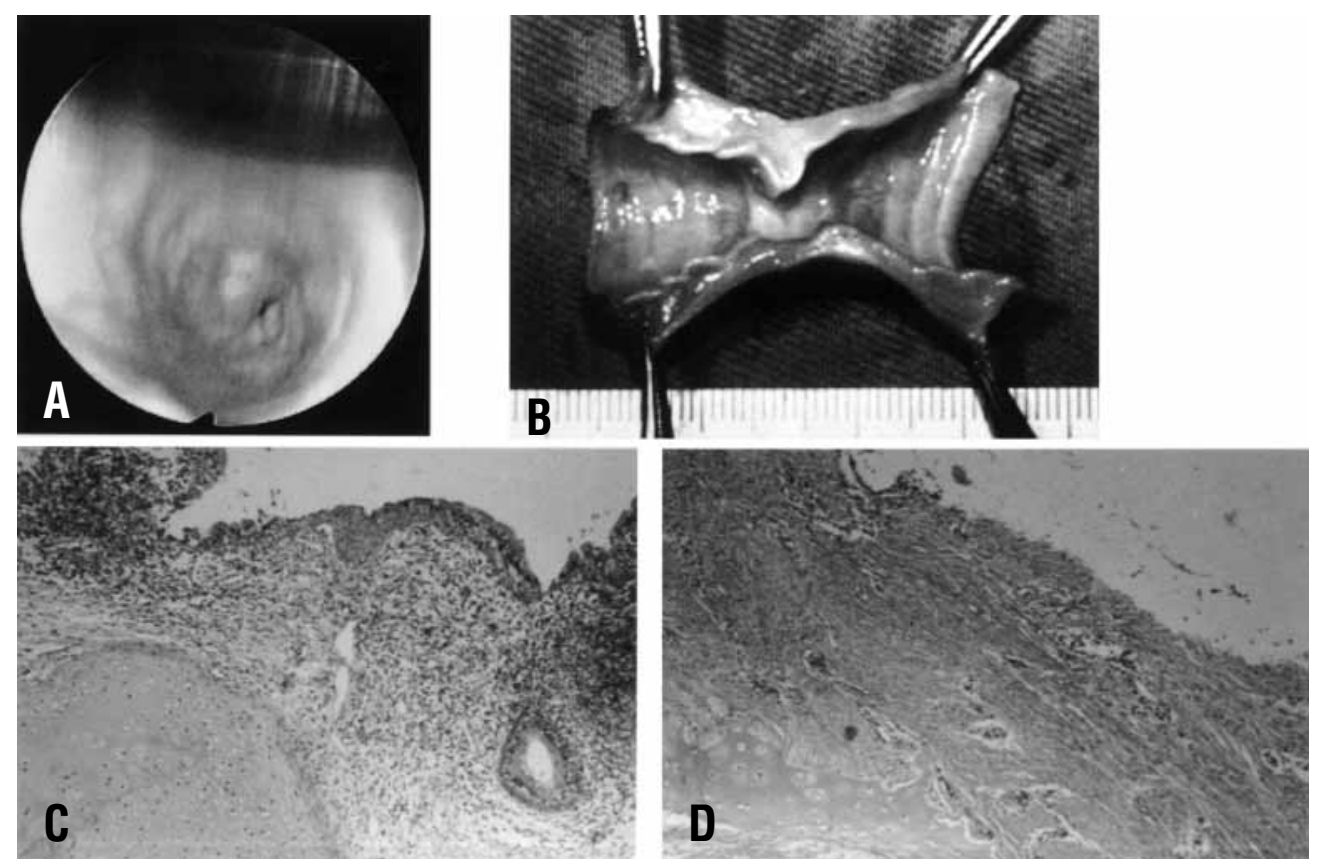

Figure 5. Bronchoscopic, macroscopic, and histologic findings of the control group. In the control group severe stenosis occurred in all animals (A and B). Histologically, remarkable cellular infiltration in the transplanted grafts of the fresh allotransplantation animals (C) and severe fibrosis and the destruction of the cartilaginous rings in the transplanted graft of the simply frozen allograft animal (D) were observed. This Figure represents findings 3 months after transplantation. Hematoxylin-and-eosin staining: original magnifications $\times 4$ (C and D).

(Figure 3,C), but the structure of the cartilaginous rings was well preserved, even after 12 months. Good epithelialization of the airway was observed (Figures 3, $D$ and $E$, and 4, A). The intracellular organelles of the epithelium appeared to be well developed, indicating proper functioning (Figure 4, B). A small degree of mononuclear cell infiltration was observed in the subepithelial layer (Figure 3, E). In all primates in this group, these findings were the same, and no progressive graft failure was observed, even after 12 months.

All the primates in the control group survived (Table 2), but severe stenosis of the transplanted graft was observed in all animals (Figure 5, $A$ and $B$ ). Slight tracheal narrowing 
TABLE 3. Results for immunohistochemical staining of tracheal tissues with anti-CD3 antibody, anti-MHC class II antibody, and anti-S100 protein antibody

\begin{tabular}{lccc}
\hline & & MHC & S100 \\
& CD3 & class II & protein \\
\hline Fresh trachea & & & \\
$\quad$ Airway epithelium & - & + & - \\
Cells in the subepithelial layer & - & - & - \\
Cryopreserved trachea & & & \\
$\quad$ Airway epithelium & - & - & - \\
$\quad$ Cells in the subepithelial layer & - & - & - \\
Trachea of the cryopreserved allotransplantation group & & \\
$\quad$ Airway epithelium & - & - & - \\
Infiltrating cells in the subepithelial layer & - & - & - \\
Trachea of the fresh allotransplantation group & & & \\
$\quad$ Airway epithelium & - & + & - \\
$\quad$ Infiltrating cells in the subepithelial layer & + & + & + \\
\hline
\end{tabular}

was observed 1 month after the operation, but a 5-mm bronchoscope could pass through the narrowing portion at this period. The degree of tracheal narrowing became severe within 2 months after the operation, and a 5-mm bronchoscope could not pass through the narrowing portion during this period. Histologically, remarkable lymphocytic and mononuclear cell infiltration and malgranulation were observed in the transplanted grafts of the fresh allotransplantation group (Figure 5, C). Severe fibrosis and the destruction of the cartilaginous rings were observed in the animal that received the simply frozen allograft (Figure 5,D).

Results of immunohistochemical staining are shown in Table 3. Fresh tracheal tissues were negative for CD3 and S100 protein immunostaining, but the presence of MHC class II antigen was identified in the airway epithelium of the fresh trachea. The cryopreserved tracheal allograft tissues were negative for $\mathrm{CD} 3$, MHC class II antigen, and S100 protein. In the cryopreserved tracheal allotransplantation group, a small degree of mononuclear cell infiltration was observed in the subepithelial layer (Figure 3,E), but these infiltrating cells were negative for CD3, MHC class II antigen, and S100 protein. In the cryopreserved tracheal allotransplantation group, the epithelial lining on the luminal surface of the allografts was negative for MHC class II antigen. In the fresh allotransplantation group, the expression of MHC class II antigen was identified in the epithelium of the allograft, and the submucosal infiltrating cells were strongly positive for $\mathrm{CD} 3$, MHC class II antigen, and S100 protein, indicating a positive immunologic reaction.

\section{Discussion}

Cryopreserved tracheal allotransplantation has been performed in various animal models, with encouraging results for clinical applications in human subjects. ${ }^{1-5}$ The problems of maintaining blood supply to the transplanted tracheal tissue, ${ }^{11-13,17}$ trachea allogenicity, ${ }^{18-23}$ and inevitable infections have been focused on in animal trials. In animal experiments cryopreserved tracheal allografts have been reported to be less immunogenic than fresh allografts, $4,5,8,24$ and infection of the transplanted trachea or mediastinitis has been well controlled with antibiotics. As Rose and colleagues, ${ }^{25}$ who performed a tracheal allotransplantation in a human patient, reported in 1979, the ischemic nature of the trachea is the most important matter of concern in tracheal allotransplantation. In animal trials on cryopreserved tracheal allotransplantation, many procedures for improving the blood supply to the trachea, such as the use of a pedicled omental flap, have been investigated. ${ }^{5}$ By applying these methods of improving the blood supply to the graft, the allotransplantation of a 5-ring length of cryopreserved trachea without the use of immunosuppressants has became possible in animal models. ${ }^{11-13}$

As described in the introduction, interspecies differences must be examined before cryopreserved tracheal allotransplantations can be safely and successfully performed in human subjects. Preservation of the blood supply to the transplanted trachea is essential for successful transplants, and graft ischemia ${ }^{11-13}$ and graft rejection ${ }^{4,5,8}$ are the main reasons for tracheal transplantation failures. As Salassa and colleagues ${ }^{26}$ pointed out, the description of the tracheal microscopic blood supply in mammals by Sobin and coworkers $^{27}$ does not apply in human subjects. Sobin and coworkers studied microcirculation in the tracheal mucosa of rabbits, dogs, rats, and guinea pigs and described marked interspecies differences in the vascular bed. Salassa and colleagues ${ }^{26}$ reported that the fine but important longitudinal anastomoses along the lateral tracheal wall and transverse vessels of the trachea were better developed in cats and dogs than in humans. Moreover, the speed of tissue regeneration in dogs is known to be faster than that in humans, as shown by the history of arterial prosthesis development. ${ }^{28}$ Most cryopreserved tracheal allotransplantation experiments have been performed with canine models. ${ }^{1,4,5,8}$ The results obtained in canine models might be modified by interspecies differences in blood supply to the trachea. Consequently, the results of cryopreserved tracheal allotransplantation experiments might be more limited than anticipated on the basis of animal models. ${ }^{26,27}$

Because tracheal tissue is especially vulnerable to ischemia, the use of cryopreserved tracheal tissue allotransplantations may be restricted. ${ }^{9-13}$ Moreover, the additional problems of rejection and contamination during tracheal reconstruction are also a concern. Thus far, many experimental models have shown that cryopreserved tracheal allografts have a lower immunogenicity than that of fresh allografts and that infectious problems arising from tracheal 
reconstruction do not occur when cryopreserved tracheal allografts are used. ${ }^{4,5,8,24}$ One important question that animal studies have yet to answer is whether cryopreserved tracheal allotransplantations are really possible in human subjects. This study with primates was performed in an attempt to answer this question. Most of the findings obtained in previous animal studies were confirmed in our study with primates. Cryopreserved allografts have a lower allogenicity than fresh allografts, partly because of the exfoliation of airway epithelium, which can act as alloantigen-presenting cells. ${ }^{18,20,22}$ The importance of programmed freezing (which augments tissue viability after cryopreservation), good airway epithelialization after tracheal allotransplantation, and good tracheal luminal patency without stenosis or the formation of malgranulation after allotransplantation were also confirmed. The long cryopreservation period did not influence the ability for a graft to take, and this result seems to support the previous report by Kushibe and colleagues. ${ }^{29}$ The most important result of our study was the fact that the cryopreserved tracheal allograftings were successful in the primates. Because improvements in tissue cryopreservation techniques have made it possible for a supply of tissues to be created, ${ }^{7}$ our successful results with primates, which are similar to humans in both anatomy and immunology, are encouraging for the clinical use of cryopreserved tracheal allografts.

In this study monthly flexible fiberoptic bronchoscopies were performed after the tracheal allotransplantation as postoperative evaluation of transplanted graft status, but biopsies were not performed because we were concerned about possible impairment of mucosa regeneration by biopsies. Instead, the date of animal death in the cryopreserved allotransplantation group was scheduled at various postoperative times (ie, short-term to long-term), as shown in Table 1, and we evaluated pathology of the transplanted grafts. Considering the pathologic findings, epithelial regeneration and mononuclear cell infiltration in the cryopreserved allotransplantation group seemed to occur within 1 month. Because slight tracheal stenosis was observed 1 month after the operation in the fresh allotransplantation group, infiltration of CD3-, MHC class II antigen-, and S100-positive cells was considered to occur within 1 month.

In this study cryopreserved allografts showed a lower allogenicity than fresh allografts; however, a small degree of mononuclear cell infiltration, indicating immunogenic rejection, was observed even in the cryopreserved allotransplantation group. High allogenicity of fresh tracheal allograft with resultant strong rejection ${ }^{4,22}$ seems to be attenuated by a short course of immunosuppressive therapy, as reported by Kawahara and colleagues. ${ }^{30}$ In cryopreserved tracheal allotransplantation, a short course of immunosuppressive therapy also might be beneficial for control of rejection.
Of course, our results were obtained with only a small number of animals. Histologic findings indicating slight immunogenic rejection, such as mononuclear cell infiltration, were observed in the cryopreserved tracheal allografts. Longer follow-up might be necessary to confirm the longterm fate of transplanted grafts. Moreover, tracheal tissue is vulnerable to ischemia, and successful tracheal allotransplantations seem to require tracheal organ transplantation, as pointed out in previous reports. ${ }^{9,10}$ However, in cases in which a small augmentation of the tracheal lumen can benefit patients with severe tracheal stenosis, cryopreserved tracheal homograft allotransplantations may be an important contribution to the patient's respiratory condition. Jacobs and colleagues ${ }^{14}$ and Elliot and coworkers ${ }^{15}$ have reported the successful management of long-segment tracheal stenosis using chemically preserved tracheal allografts, and Kunachak and colleagues ${ }^{16}$ have reported a successful short-segment laryngotracheal reconstruction using a cryopreserved tracheal allograft. Their reports showed that long-segment ${ }^{14,15}$ and short-segment ${ }^{16}$ patch trachoplasty with a tracheal allograft could be applicable and beneficial for selected patients. In our preliminary study we tried short-segment reconstruction, using their method with encouraging results. Short-segment tracheal reconstruction using our method seems to be applicable for human subjects. However, there may be a limit to the length of reconstruction, as shown by previous studies. ${ }^{11-13}$ In our future studies long-segment reconstruction with our method should be tried because most short segments can be primarily reconstructed. Further investigation of the possible clinical applications of cryopreserved tracheal allografts in human subjects will be important in the future.

\section{Conclusion}

Experimental cryopreserved tracheal allotransplantation was successful in a primate model. Possible clinical applications of cryopreserved tracheal allografts in human subjects and the limitations of cryopreserved tracheal allografting should be investigated in the future.

We thank Mr Nobutaka Furuya, Mr Takashi Kubota, and Ms Noriko Yoneda for their assistance with this experiment.

\section{References}

1. Ueda M, Yokomise H, Wada H, Hitomi S. Experimental tracheal transplantation for possible clinical application. Transplant Proc. 1997;29:871-3.

2. Aoki T, Yamato Y, Tsuchida M, Souma T, Yoshiya K, Watanabe T, et al. Successful tracheal transplantation using cryopreserved allografts in a rat model. Eur J Cardiothorac Surg. 1999;16:169-73.

3. Yokomise H, Inui K, Wada H, Ueda M, Hitomi S. Long-term cryopreservation can prevent rejection of canine tracheal allografts with preservation of graft viability. J Thorac Cardiovasc Surg. 1996;111: 930-4.

4. Tojo T, Niwaya K, Sawabata N, Kushibe K, Nezu K, Taniguchi S, et al. Tracheal replacement with cryopreserved tracheal allograft: experiment in dogs. Ann Thorac Surg. 1998;66:209-13. 
5. Mukaida T, Shimizu N, Aoe M, Andou A, Date H, Okabe K, et al. Experimental study of tracheal allotransplantation with cryopreserved grafts. J Thorac Cardiovasc Surg. 1998;116:262-6.

6. Hoekstra FM, Witvliet M, Knoop CY, Wassenaar C, Bogers AJ, Weimar W, et al. Immunogenic human leukocyte antigen class II antigens on human cardiac valves induce specific alloantibodies. Ann Thorac Surg. 1998;66:2022-6.

7. Hopkins RA. Cardiac reconstructions with allograft valve. New York: Springer-Verlag; 1989. p. 55-8.

8. Tojo T, Niwaya K, Sawabata N, Nezu K, Kawachi K, Kitamura S. Tracheal allogenic immunoresponse is reduced by cryopreservation: canine experiment. Transplant Proc. 1996;28:1814-5.

9. Lenot B, Macchiarini P, Dulmet E, Weiss M, Dartevell P. Tracheal allograft replacement: an unsuccessful method. Eur J Cardiothorac Surg. 1993;7:648-52.

10. Macchiarini P, Mazmanian GM, de Montpreville V, Dulmet E. Fatta M, Lenot B, et al. Experimental tracheal and tracheoesophageal allotransplantation. J Thorac Cardiovasc Surg. 1995;110:1037-46.

11. Yokomise $\mathrm{H}$, Inui $\mathrm{K}$, Wada $\mathrm{H}$, Hitomi $\mathrm{S}$. The infeasibility of using tenring irradiated grafts for tracheal allotransplantation even with omentopexy. Surg Today. 1996;26:427-30.

12. Nakanishi R, Shirakusa T, Mitsudomi T. Maximal length of tracheal autografts in dogs. J Thorac Cardiovasc Surg. 1993;106:1081-7.

13. Yokomise H, Inui K, Wada H, Ueda M, Hitomi S, Itoh H. Split transplantation of the trachea: a new operative procedure for extended tracheal resection. J Thorac Cardiovasc Surg. 1996;112:314-8.

14. Jacobs JP, Quintessenza JA, Andrews T, Burke RP, Spektor Z, Delius RE, et al. Tracheal allograft reconstruction: the total north American and worldwide pediatric experience. Ann Thorac Surg. 1999;68:1043-52.

15. Elliot MJ, Haw MP, Jacobs JP, Bailey CM, Evans JNG, Herberhold C. Tracheal reconstruction in children using cadaveric homograft trachea. Eur J Cardiothorac Surg. 1996;10:707-12.

16. Kunachak S, Kulapaditharom B, Vajaradul Y, Rochanawutanon M. Cryopreserved, irradiated tracheal homograft transplantation for laryngotracheal reconstruction in human beings. Otolaryngol Head Neck Surg. 2000;122:911-6.

17. Deschamps C, Trastek VF, Ferguson JL, Martin WJ, Colby TV, Pairolero PC, et al. Cryopreservation of canine trachea: functional and histological changes. Ann Thorac Surg. 1989;47:208-12.
18. Nakajima J, Ono M, Takeda M, Kawauchi M, Furuse A, Takizawa $\mathrm{H}$. Role of costimulatory molecules on airway epithelial cells acting as alloantigen-presenting cells. Transplant Proc. 1997;29:2297300 .

19. Shaari CM, Farber D, Brandwein MS, Gannon P, Urken ML Characterizing the antigenic profile of the human trachea: implication for tracheal transplantation. Head Neck. 1998;20:522-7.

20. Lane BP, Habicht GS, Jasper GS. Lymphocyte-epithelium interaction during rejection of nonisogeneic rat tracheal grafts. Am J Pathol. 1977;86:71-80.

21. Bujia J, Wilmes E, Hammer C, Kastenbauer E. Tracheal transplantation: demonstration of HLA class I subregion gene products on human trachea. Acta Otolaryngol. 1990;110:149-54.

22. Liu Y, Nakamura T, Yamamoto Y, Matsumoto K, Sekine T, Ueda H, et al. Immunosuppressant-free allotransplantation of the trachea: the antigenicity of tracheal grafts can be reduced by removing the epithelium and mixed glands from the graft by detergent treatment. J Thorac Cardiovasc Surg. 2000;120:108-14.

23. Nakanishi R, Shirakusa T, Hanagiri T. Early histopathologic features of tracheal allotransplant rejection: a study in nonimmunosuppressed dogs. Transplant Proc. 1994;26:3715-8.

24. Moriyama H, Sasajima T, Hirata S, Yamazaki K, Yatsuyanagi E, Kubo Y. Revascularization of canine cryopreserved tracheal allografts. Ann Thorac Surg. 2000;69:1701-6.

25. Rose KG, Sesterhenn K, Wustrow F. Tracheal allotransplantation in man [letter]. Lancet. 1979;1:433.

26. Salassa JR, Pearson BW, Payne WS. Gross and microscopical blood supply of the trachea. Ann Thorac Surg. 1977;24:100-7.

27. Sobin SS, Frasher WG, Tremer HM, Hadley GG. The microcirculation of the tracheal mucosa. Angiology. 1963;14:165-9.

28. Sauvage LR, Berger KE, Wood SJ, Yates SG, Smith JC, Mansfield PB. Interspecies healing of porous arterial protheses: observations, 1960 to 1974. Arch Surg. 1974;109:698-705.

29. Kushibe K, Nezu K, Nishizaki K, Takahama M, Taniguchi S. Tracheal allotransplantation maintaining cartilage viability with long-term cryopreserved allografts. Ann Thorac Surg. 2001;71:1666-9.

30. Kawahara K, Inutsuka K, Hiratsuka M, Makihata S, Okabayashi K, Shiraishi T, et al. Tracheal transplantation for carinal reconstruction in dogs. J Thorac Cardiovasc Surg. 1998;116:397-401. 